\title{
Slicing the genome of star-fruit (Averrhoa carambola L.)
}

3

4

11 Running title: Draft genome of the star fruit

\section{Authors} Xin $\mathrm{Liu}^{1}$, Huan $\mathrm{Liu}^{1,4^{*}}$

\#These authors contributed equally

*Corresponding author

\section{Abstract} fruit plant. sequencing, phylogenetic tree

\section{Introduction}

Yannan Fan ${ }^{1 \#}$, Sunil Kumar Sahu ${ }^{1 \#}$, Ting Yang ${ }^{1}$, Weixue $\mathrm{Mu}^{1}$, Jinpu Wei ${ }^{1}$, Le Cheng ${ }^{2}$, Jinlong Yang ${ }^{2}$, Ranchang $\mathrm{Mu}^{3}$, Jie $\mathrm{Liu}^{3}$, Jianming Zhao ${ }^{3}$, Yuxian Zhao ${ }^{5}$, Xun $\mathrm{Xu}^{1,6}$,

The Averrhoa carambola is commonly known as star fruit because of its peculiar shape and its fruit is a rich source of minerals and vitamins. It is also used in traditional medicines in countries like India, China, the Philippines, and Brazil for treating various ailments such as fever, diarrhea, vomiting, and skin disease. Here we present the first draft genome of the Oxalidaceae family with an assembled genome size of $470.51 \mathrm{Mb}$. In total, 24,726 protein-coding genes were identified and 16,490 genes were annotated using various well-known databases. The phylogenomic analysis confirmed the evolutionary position of the Oxalidaceae family. Based on the gene functional annotations, we also discovered the enzymes possibly involved in the important nutritional pathways in star fruit genome. Overall, being the first sequenced genome in the Oxalidaceae family, the data provides an essential resource for the nutritional, medicinal, and cultivational studies for this economically important star-

Keywords: star-fruits, medicinal plant, Averrhoa carambola L., whole-genome

The star-fruit plant (Averrhoa carambola L.), a member of the Oxalidaceae family, is a medium-sized tree which is distinguished for its unique and attractive star-shaped fruit (Supplementary Fig.1). A. carambola is widely distributed around the world, especially in tropical countries, such as India, Malaysia, Indonesia, and the Philippines and is considered as an important species, and thus it is extensively cultivated in South- 
east Asia and Malaysia 1,2. Besides, it is also a popular fruit in the United States, Australia, and the South Pacific Islands market ${ }^{3}$. Star-fruits have a unique taste with slightly tart, acidic (smaller fruits) and sweet, mild flavor (large fruits). The star-fruit is known as a good source of various minerals and vitamins, and it is also rich in natural antioxidants such as vitamin $\mathrm{C}$ and gallic acid. Moreover, the presence of high amounts of fibers in fruits aids in absorbing glucose and retarding the glucose diffusion into the bloodstream and helps in controlling blood glucose concentration.

In addition to the food sources, it is also utilized as herbs in India, Brazil, and Malaysia, and it is widely used in traditional Chinese Medicine preparations ${ }^{4}$, as a remedy for fever, asthma, headache, and skin diseases ${ }^{5}$. Several studies have demonstrated the presence of various phytochemicals such as saponins, flavonoids, alkaloids and tannins in the leaves, fruits, and roots of star-fruit plant ${ }^{6,7}$, which are known to confer antioxidant and specific healing properties. The study of Cabrini et al., 5 indicated that the ethanolic extract from $A$. carambola is highly useful in minimizing the symptoms of ear swelling (edema) and cellular migration in mice. The flavonoid compound (apigenin-6-C- $\beta$-fucopyranoside), which isolated from $A$. carambola leaves, showed the anti-hyperglycemic action in rats, and this might be a potential treatment and prevention of diabetes ${ }^{8}$. Moreover, the DMDD (2-dodecyl-6-methoxycyclohexa2,5-diene-1,4-dione) extracted from the root of $A$. carambola exhibits potential benefits against obesity, insulin resistance, and memory deficits in Alzheimer's disease ${ }^{9,10}$.

Even though $A$. carambola plays very significant roles in traditional medicine appalments, there are very limited studies at the genetic level on $A$. carambola., mainly due to the lack of genome information. Therefore, filling this genomic gap will help the researchers to fully explore and understand this agriculturally important plant. As a part of 10KP project ${ }^{11,12}$, in this study, the draft genome of $A$. carambola collected from Ruili botanical garden, Yunnan, China was assembled using advanced 10X genomics technique to further understand the evolution of Oxalidaceae family. Furthermore, a fully annotated genome of $A$. carambola would serve as a foundation for the pharmaceutical applications and in the improvement of breeding strategies of the starfruit plant.

\section{Results}

\section{Genome assembly and evaluation}

Based on the k-mer analysis, the total number of 35,655,285,391 kmers were used, with a peak coverage is 75 . The $A$. carambola genome was estimated to be $\sim 475 \mathrm{Mb}$ in size (Supplementary Fig. 2). To perform the assembly, a total of $156 \mathrm{~Gb}$ clean reads were utilized by Supernova v2.1.1 ${ }^{13}$. The final assembly contained 69,402 scaffold sequences with $\mathrm{N} 50$ of $2.76 \mathrm{Mb}$ and 78,313 contig sequences with $\mathrm{N} 50$ of $44.84 \mathrm{~Kb}$ for a total assembly size of $470.51 \mathrm{Mb}$ (Table 1). Completeness assessment was performed using BUSCO (Bench-marking Universal Single-Copy Orthologs) version 3.0.1 ${ }^{14}$ with 
Embryophyta odb9. The result showed that 1327 (92.20\%) of the expected 1440 conserved plant orthologs were detected as complete (Supplementary Table 1). In order to further evaluate the completeness of the assembled genome, we performed short reads mapping using clean raw data by BWA-MEM software ${ }^{15}$. In total, $943,278,896$ (99.12\%) reads can be mapped to the genome and $88.13 \%$ of them were properly paired (Supplementary Table 2).

Table 1. Statistics of genome assembly

\section{Contig}

Scaffold

\begin{tabular}{cllll} 
& Size(bp) & Number & Size(bp) & Number \\
\hline N90* & 5,420 & 12,457 & 7,033 & 3,988 \\
\hline N80 & 13,875 & 7,548 & 39,210 & 608 \\
\hline N70 & 23,325 & 5,165 & 34,6109 & 131 \\
\hline N60 & 33,460 & 3,619 & $1,307,770$ & 60 \\
\hline N50 & 44,841 & 2,503 & $2,757,598$ & 35 \\
\hline Longest & 717,770 & - & $14,768,062$ & - \\
\hline Total Size & $431,262,337$ & - & $470,508,511$ & - \\
\hline $\begin{array}{c}\text { Total number } \\
\text { (>=2kb) }\end{array}$ & - & 18,820 & - & 10,777 \\
\hline
\end{tabular}

Total number - $\quad 78,313 \quad$ - $\quad 69,402$

(>=100bp)

*: Nxx length is the maximum length $\mathrm{L}$ such that $\mathrm{xx} \%$ of all nucleotides lie in contigs (or scaffolds) of size at least L.

\section{Genome annotation}

A total of $68.15 \%$ of the assembled $A$. carambola genome was composed of repetitive elements (Supplementary Table 3). Among these repetitive sequences, the LTRs were the most abundant, accounting for $61.64 \%$ of the genome. DNA class repeat elements represented $4.19 \%$ of the genome; LINE and SINE classes encoded $0.28 \%$ and $0.016 \%$ of the assembled genome, respectively. For the gene prediction, we combined homology- and de novo- based approaches and obtained a non-redundant set of 24,726 gene models with 4.11 exon per gene on average. The gene length was 3,457 bp on average, while the average exon and intron lengths were $215 \mathrm{bp}$ and $827 \mathrm{bp}$, respectively. The gene model statistics data compared with the other seven homology species are shown in Supplementary Fig 3. To evaluate the completeness of the gene models for $A$. carambola, we used BUSCO with Embryophyta odb9. There are 1281 $(88.9 \%)$ complete orthologs were detected by the star fruit predicted gene sets 
(Supplementary Table 4).

Functions were assigned to $16,490(66.69 \%)$ genes. These protein-coding genes were then subjected for further exploration against KEGG, NR and COGs protein sequence databases ${ }^{16}$, in addition to SwissProt, and TrEMBL ${ }^{17}$, and then InterProScan 18 was lastly used to identify domains and motifs (Supplementary Table 5, Supplementary Fig. 4). Non-coding RNA genes in the assembled genome were also annotated. We predicted 759 tRNA, 1,341 rRNA, 90 microRNA (miRNA) and 2,039 small nuclear RNA (snRNA) genes in the assembled genome (Supplementary Table 6).

Since star fruit is an important cultivated plant, the identification of diseaseresistance genes was one of the focus in our study. The NBS (nucleotide-binding site) genes play an important role in pathogen defense and cell cycle. We identified a total of 80 non-redundant NBS-encoding orthologous genes in the star fruit genome (Supplementary Table 7). Among those genes, the TIR (encoding the toll interleukin receptor) motif was found to be significantly smaller than other eudicot plants, except cocoa. Unlike other plants, the LRR (Leucine rich repeat) motif in star fruit is not the most or second most type in NBS gene family ${ }^{19}$.

\section{Genome evolution}

120 The characterization of star fruit genome can provide the necessary features to further analyze the evolutionary history of Oxalidaceae. The $\gamma$ whole genome triplication event represents over $75 \%$ of extant angiosperms, and is associated with the early diversification of the core eudicots. In order to investigate the evolutionary events at the genomic level in star fruit, we identified 1,134 paralogous gene families on the basis of the 24,726 gene models. The synonymous substitution rates (Ks) in the duplicated genes $(\mathrm{Ks}=1.9)$ suggested that an ancient $\gamma$ event occurred in star fruit (Fig. 1a).

127 Furthermore, we performed an intergenomic collinearity between Arabidopsis 20, 128 Poplar ${ }^{21}$, and grape 22 genomes and identified relationships among star fruit 129 orthologous. The mean Ks for the one-to-one orthologous analysis of star fruit to 130 Arabidopsis, Poplar, and grape was 1.8, 1.0, and 1.2, respectively (Fig. 1b-d). The 131 result confirmed the shared $\gamma$ WGD event between the four species. Moreover, we also 132 generated the whole-genome syntenic dotplots of star fruit based on the Ks value (Fig.

133 1e). Over $50 \%$ of the syntenic blocks shared the Ks rate between 1.0 to 2.0, and only 134 about $10 \%$ of the gene pairs shared the Ks under 1.0, which indicated that no recent WGDs occurred in the star fruit genome. 

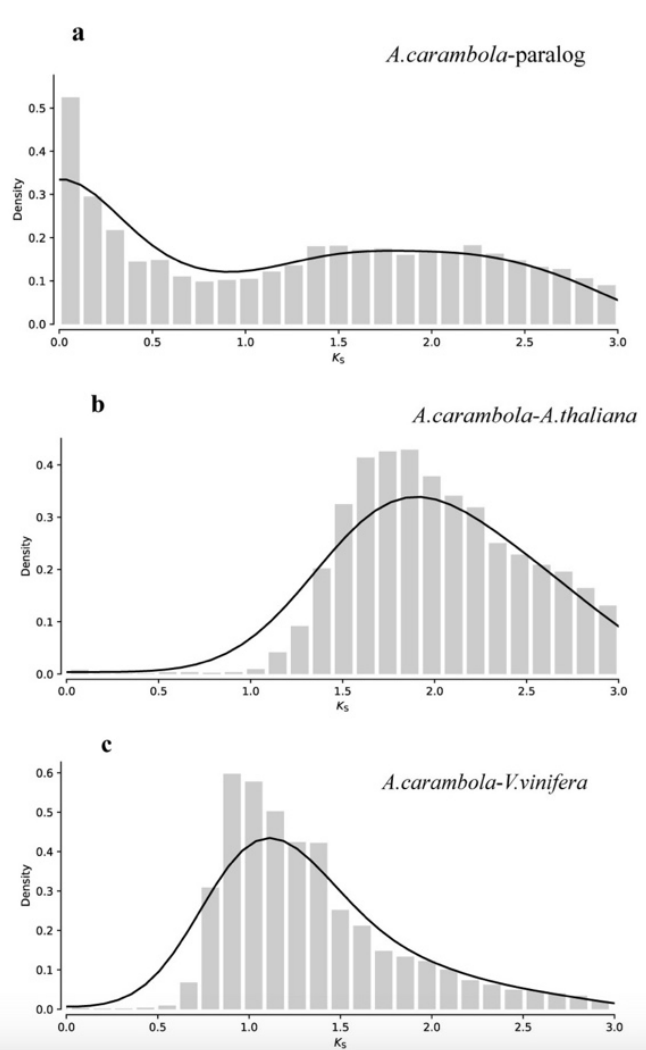
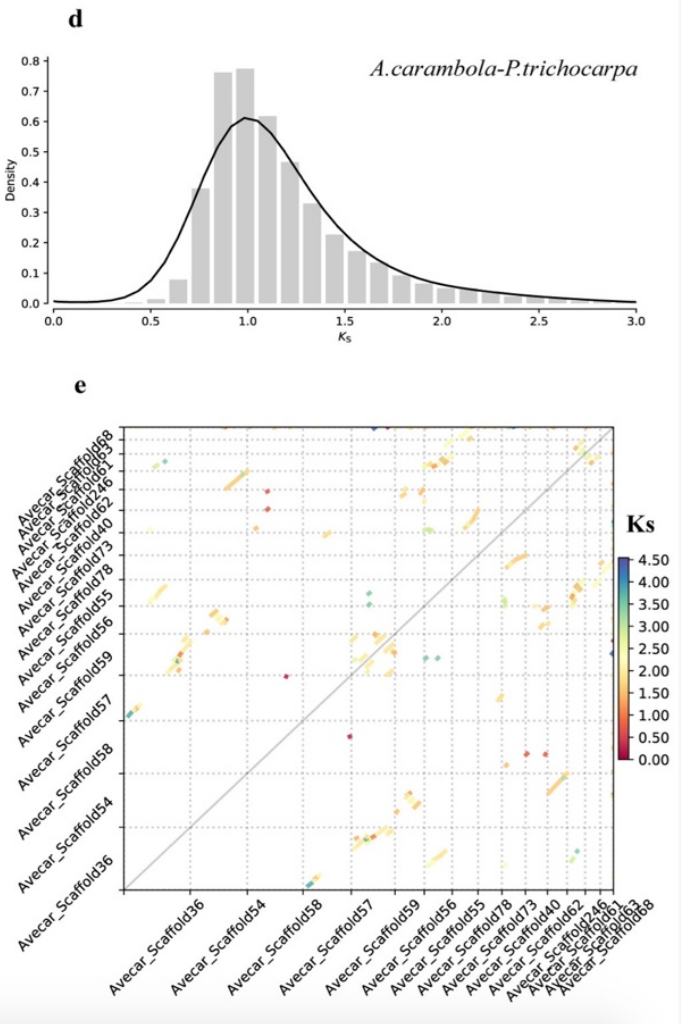

Figure 1. The $A$. carambola genome duplication. The distribution of synonymous substitution rate (Ks) distance values observed for (a) A. carambola-paralog, (b) $A$. carambola-A. thaliana ortholog, (c) A. carambola- $V$. vinifera ortholog, and (d) A. carambola-P. trichocarpa ortholog. (e) The colinear blocks of $A$. carambola paralog colored by Ks value.

\section{Gene family analysis and phylogenetic tree}

We performed $A$. carambola gene family analysis using OrthoMCL software ${ }^{23}$, with protein and nucleotide sequences from $A$. carambola and ten other plant species $(A$. thaliana, C. sinensis, F. sylvatica, G. max, K. fedtschenkoi, M. domestica, P. granatum, P. trichocarpa, T. cacao, V. vinifera) based on an all-versus-all BLASTP alignment with an E-value cutoff of 1e-05. The predicted 24,726 protein-coding genes in A. carambola were assigned to 9,731 gene families consisting of 15,301 genes, while 9,425 were not organized into groups, which were unique for A. carambola (Supplementary Table 8, Fig. 2b). In total, 163 single-copy orthologs corresponding to the eleven species were extracted from the clusters and were used to construct the phylogenetic tree. The constructed tree topology supported the APG IV ${ }^{24}$ system that Oxalidales $(A$. carambola) and Malpighiales (P. trichocarpa) are sister clades which belong to the same cluster Rosids. Based on the phylogenetic tree, A. carambola was estimated to separate from P. trichocarpa, $V$. vinifera and $K$. fedtschenkoi approximately 94.5, 110.2 and 126.3 Mya, respectively (Supplementary Fig. 5).

We also analyzed the expansion and contraction of the gene families between species using CAFÉ. The result showed that 888 gene families were substantially 
161 expanded and 15,724 gene families were contracted in A carambola (Fig. 2a). In total, 1622,916 and 6,057 genes of $A$. carambola were identified from expanded and contracted 163 families, respectively. which the contraction was about 2 times more than expansion.

164 Later, the Gene Ontology (GO) and KEGG functional enrichment analyses were performed for all expansion gene families. The KEGG pathway enrichment analysis results are shown in Table 2 and the GO enrichment are listed in Supplementary Table 9. In a previous study, researchers had isolated the flavonoids from the fresh fruit of $A$. carambola, which are known to reduce the harmful inflammation ${ }^{25}$. In our study, the flavonoid biosynthesis pathway was found to be significantly enriched in the expanded families. Terpenoids are yet another important type of compound which has been isolated from star fruit ${ }^{26}$, which has been proven to exhibit anti-inflammatory activities. A. carambola likely synthesize terpenoids via the Diterpenoid biosynthesis pathway.

a

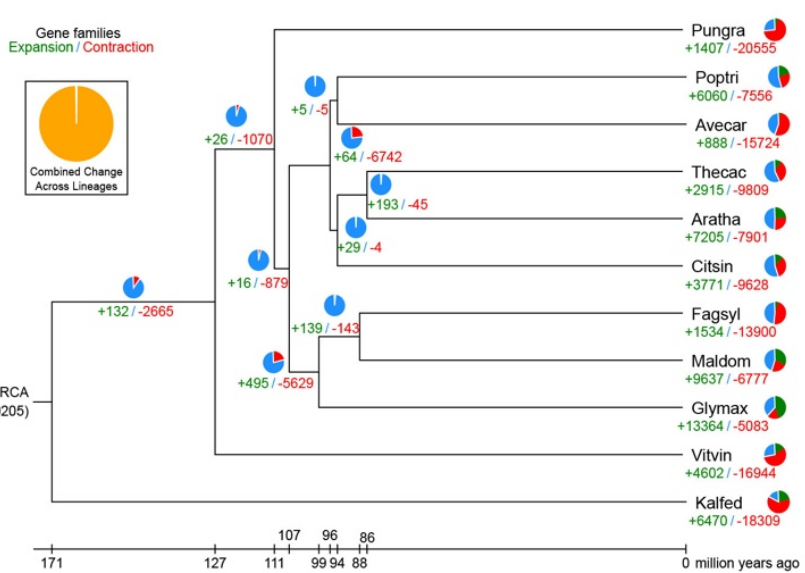

b

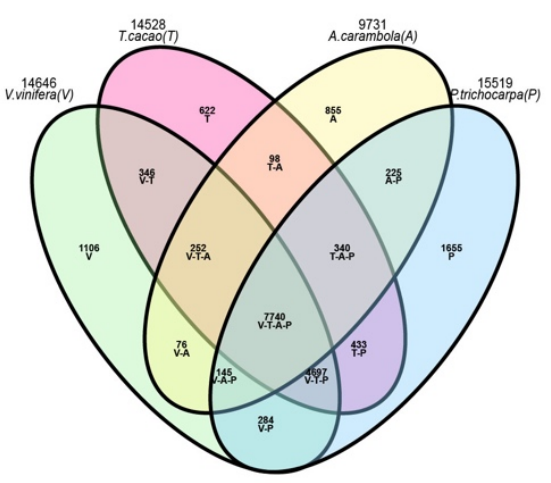

Figure 2. Gene family analysis and phylogenetic tree construction. (a) The

176 phylogenetic tree with the expansion and contraction size of gene families. Pungra: $P$.

177 granatum; Poptri: P. trichocarpa; Avecar: A. carambola; Thecac: T. cacao; Aratha: A.

178 thaliana; Citsin: C. sinensis; Fagsyl: F. sylvatica; Maldom: M. domestica; Glymax:

179 G. max; Vitvin: V. vinifera; Kalfed: K. fedtschenkoi. (b) The Venn diagram for the

180 number of shared gene families within A. carambola, $V$. vinifera, $T$. cacao and $P$.

181 trichocarpa.

182

183 Table 2. Enriched KEGG pathways of unique genes of $\boldsymbol{A}$. carambola with 184 expansion. 185

\begin{tabular}{lllll}
\hline Pathway ID & KEGG description & $\begin{array}{l}\text { Adjusted } \\
\text { P-value } \\
(<=\mathbf{0 . 0 5})\end{array}$ & $\begin{array}{l}\text { Number } \\
\text { of genes }\end{array}$ & $\begin{array}{l}\text { Total genes } \\
\text { in the } \\
\text { pathway }\end{array}$ \\
\hline map03020 & RNA polymerase & $3.51 \mathrm{E}-09$ & 30 & 137 \\
map00904 & Diterpenoid biosynthesis & $1.00 \mathrm{E}-05$ & 18 & 63 \\
map00240 & Pyrimidine metabolism & $3.16 \mathrm{E}-05$ & 34 & 215
\end{tabular}




$\begin{array}{lllll}\text { map01100 } & \text { Metabolic pathways } & 7.17 \mathrm{E}-05 & 234 & 2621 \\ \text { map00901 } & \text { Indole alkaloid biosynthesis } & 7.17 \mathrm{E}-05 & 15 & 55 \\ \text { map00230 } & \text { Purine metabolism } & 7.17 \mathrm{E}-05 & 36 & 244 \\ \text { map00565 } & \text { Ether lipid metabolism } & 0.00014092 & 14 & 52 \\ \text { map01110 } & \text { Biosynthesis of secondary metabolites } & 0.00038039 & 142 & 1478 \\ \text { map02010 } & \text { ABC transporters } & 0.00080624 & 20 & 119 \\ \text { map00902 } & \text { Monoterpenoid biosynthesis } & 0.00116462 & 9 & 29 \\ \text { map00460 } & \text { Cyanoamino acid metabolism } & 0.00270665 & 18 & 110 \\ \text { map00941 } & \text { Flavonoid biosynthesis } & 0.03063434 & 17 & 120 \\ \text { map00190 } & \text { Oxidative phosphorylation } & 0.0354555 & 19 & 142 \\ \text { map00940 } & \text { Phenylpropanoid biosynthesis } & 0.0354555 & 32 & 280 \\ \text { map00062 } & \text { Fatty acid elongation in mitochondria } & 0.0354555 & 7 & 32 \\ \text { map00563 } & \text { Glycosylphosphatidylinositol(GPI)-anchor } & 0.03937265 & 8 & 41 \\ \text { map00040 } & \text { biosynthesis } & & & 167\end{array}$

\section{Genes specially involved in star fruit nutrition pathways}

188 The star-fruit is an excellent source of various minerals and vitamins, especially for 189 natural antioxidants such as L-ascorbic acid (vitamin C) and riboflavin (vitamin $\mathrm{B}_{2}$ ) 1,25 .

190 Through the ortholog search in the KEGG pathway, we identified the enzymes which

191 are potentially involved in the vitamin $\mathrm{C}$ and vitamin $\mathrm{B}_{2}$ biosynthesis pathways in $A$.

192 carambola.

193 In a previous report, a major component of plant ascorbate was reported to 194 synthesize through the 1-galactose pathway ${ }^{27}$, in which GDP-d-mannose is converted 195 to l-ascorbate by four successive intermediates, as summarized in Fig. 3a. Laing et al. $196{ }^{28}$ reported the identification of 1-galactose guanylyltransferase encoding homologous 197 genes from Arabidopsis and kiwifruit which catalyzes the conversion of GDP-1198 galactose to 1-galactose-1-P. In this study, five necessary enzymes - GalDH, GalLDH, 199 GGalPP, GalPP, and GME were identified, which are involved in the vitamin C pathway, 200 suggesting the possibility of ascorbic acid synthesis in the star-fruit (Table 3). For the 201 L-galactose dehydrogenase (GalDH) gene, we identified 4 paralogous genes in the star 202 fruit genome. The copy number of the GalDH genes in star fruit is close to the tomato 203 (5) and papaya (4), but nearly half of other species (10 in poplar, 11 in orange, 8 in 204 Arabidopsis, and 13 in grape, Supplementary Table 12). Further evolutionary analysis 205 showed three clusters in the phylogenetic tree, and the most ancient cluster comprised 206 all the grape genes. In the other two sister clusters, one is ancient from poplar including 2074 genes, the other is closer to orange including 7 genes. The 4 genes in star fruit are 208 divided into two clusters and were recently separated from their ancestors (Fig. 3b). 
a

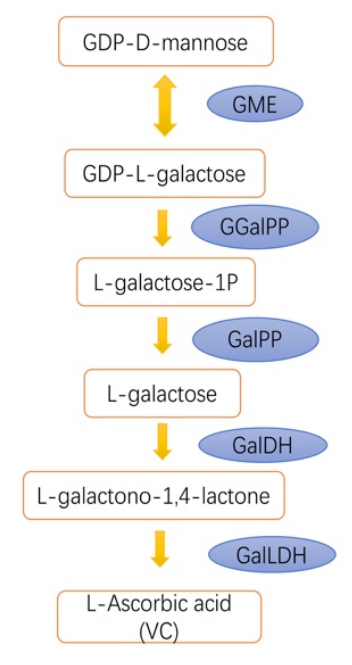

b

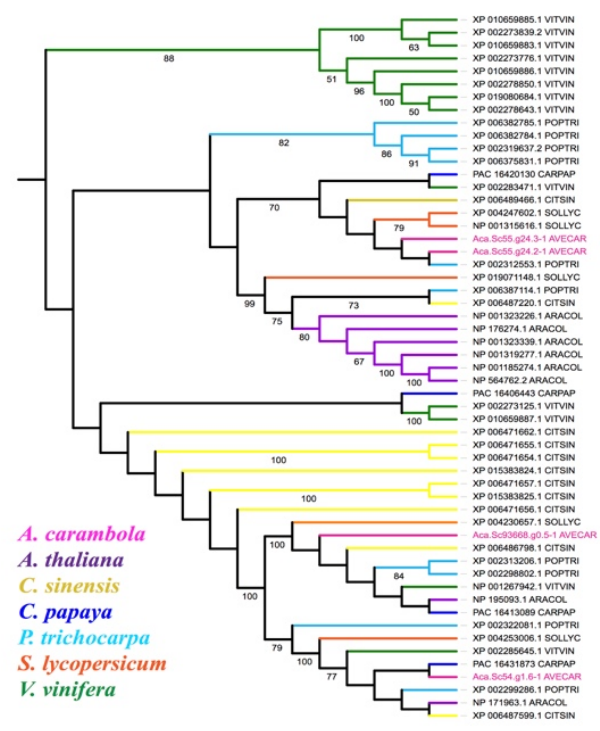

211

212

213

214

215

216

217

218

219

220

221

222

Figure 3. Genes involved in vitamin $\mathbf{C}$ metabolism. (a) A proposed model for Lascorbic acid biosynthesis pathways in star fruit. Genes identified as being involved in the pathways shown in the blue circle. (b) Phylogenetic analysis of the GalDH gene family in A. carambola (rose red), A. thaliana (purple), C. sinensis (yellow), $C$. papaya (blue), P. trichocarpa (sky blue), S. lycopersicum (orange), and V. vinifera (green).

GalDH: L-galactose dehydrogenase; GalLDH: L-galactono-1,4-lactone dehydrogenase; GGalPP: GDP-L-galactose phosphorylase; GalPP: L-galactose-1phosphate phosphatase; GME: GDP-D-mannose-3',5'-epimerase.

We also identified the possible enzymes involved in riboflavin (vitamin $\mathrm{B}_{2}$ ) biosynthesis pathway in the star-fruit (Fig. 4a, Table 3). Through the catalyzation by RIB3, RIB4, and RIB5, the D-Ribulose 5-phosphate compound can finally produce the riboflavin. Furthermore, to investigate the possible biosynthesis pathway for the special product oxalate in star fruit, we identified three enzymes - CS (citrate synthase), aceA (isocitrate lyase), and ACO (aconitate hydratase) which can potentially catalyze oxalacetic acid to glyoxylate within the glyoxylate and dicarboxylate metabolism pathway (Supplementary Table 10).

In the $A$. carambola gene family analysis, the KEGG pathway enrichment analysis for the expanded gene families revealed that 17 genes participate in flavonoid synthesis pathway $(\mathrm{P}$-value $=0.03$, Table 3). The previous studies proved that flavonoids can be isolated from A. carambola and other plants from the Oxalidaceae family 1,9,25,29-32. Here, we identified 11 enzymes that could be potentially involved in the flavonoid biosynthesis pathway (Fig. 4b, Supplementary Table 11) The most two enzymes in the pathway which contain 23 and 21 genes are HCT (shikimate O- 
237 hydroxycinnamoyltransferase) and CHS (chalcone synthase), respectively. Among the

238 end-point products, apigenin, cyanidin, epicatechin, and quercetin has been extracted

239 from the leaves, fruits or barks of A. carambola in previous studies ${ }^{6,33-35}$.

240

241 Table 3. List of genes involved in vitamin $\mathbf{C}$ and Vitamin $\mathbf{B}_{2}$ pathway 242

\begin{tabular}{|c|c|c|c|c|c|}
\hline pathway & Enzyme & Description & $\begin{array}{l}\text { Copy } \\
\text { numbers }\end{array}$ & Gene ID & $\begin{array}{l}\text { Protein } \\
\text { (AA) }\end{array}$ \\
\hline \multirow{16}{*}{$\begin{array}{l}\text { Vitamin C } \\
\text { pathway }\end{array}$} & \multirow{4}{*}{ GalDH } & \multirow{4}{*}{ L-galactose dehydrogenase } & \multirow{4}{*}{4} & Aca.sc093668.g0.5 & 332 \\
\hline & & & & Aca.sc000054.g1.6 & 334 \\
\hline & & & & Aca.sc000055.g24.2 & 351 \\
\hline & & & & Aca.sc000055.g24.3 & 594 \\
\hline & \multirow{5}{*}{ GalLDH } & \multirow{5}{*}{$\begin{array}{l}\text { L-galactono-1,4-lactone } \\
\text { dehydrogenase }\end{array}$} & \multirow{5}{*}{5} & Aca.sc000059.g19.35 & 601 \\
\hline & & & & Aca.sc000229.g0.29 & 603 \\
\hline & & & & Aca.sc000078.g43.5 & 598 \\
\hline & & & & Aca.sc100684.g0.3 & 99 \\
\hline & & & & Aca.sc102574.g0.3 & 99 \\
\hline & \multirow{3}{*}{ GGalPP } & \multirow{3}{*}{$\begin{array}{l}\text { GDP-L-galactose } \\
\text { phosphorylase }\end{array}$} & \multirow{3}{*}{3} & Aca.sc150475.g0.5 & 275 \\
\hline & & & & Aca.sc150564.g0.4 & 288 \\
\hline & & & & Aca.sc098705.g0.4 & 410 \\
\hline & \multirow{2}{*}{ GalPP } & \multirow{2}{*}{$\begin{array}{l}\text { L-galactose-1-phosphate } \\
\text { phosphatase }\end{array}$} & \multirow{2}{*}{2} & Aca.sc006151.g1 & 181 \\
\hline & & & & Aca.sc000246.g13.57 & 360 \\
\hline & \multirow{2}{*}{ GME } & \multirow{2}{*}{$\begin{array}{l}\text { GDP-D-mannose-3',5'- } \\
\text { epimerase }\end{array}$} & \multirow{2}{*}{2} & Aca.sc096116.g0.5 & 383 \\
\hline & & & & Aca.sc000061.g13.42 & 383 \\
\hline \multirow{7}{*}{$\begin{array}{l}\text { Vitamin } B_{2} \\
\text { pathway }\end{array}$} & \multirow[t]{3}{*}{ RIB3 } & \multirow{3}{*}{$\begin{array}{l}\text { 3,4-dihydroxy 2-butanone } 4 \text { - } \\
\text { phosphate synthase }\end{array}$} & \multirow{3}{*}{3} & Aca.sc000063.g38.15 & 545 \\
\hline & & & & Aca.sc000056.g65.27 & 572 \\
\hline & & & & Aca.sc000071.g11.34 & 590 \\
\hline & RIB4 & $\begin{array}{l}6,7 \text {-dimethyl-8- } \\
\text { ribityllumazine synthase }\end{array}$ & 1 & Aca.sc023103.g76.2 & 185 \\
\hline & RIB5 & riboflavin synthase & 1 & Aca.sc000036.g2.16 & 343 \\
\hline & RFK & $\begin{array}{l}\text { riboflavin } \\
\text { phosphotransferase }\end{array}$ & 1 & Aca.sc000058.g77.42 & 539 \\
\hline & FLAD1 & FMN adenylyltransferase & 1 & Aca.sc000058.g25.58 & 520 \\
\hline
\end{tabular}




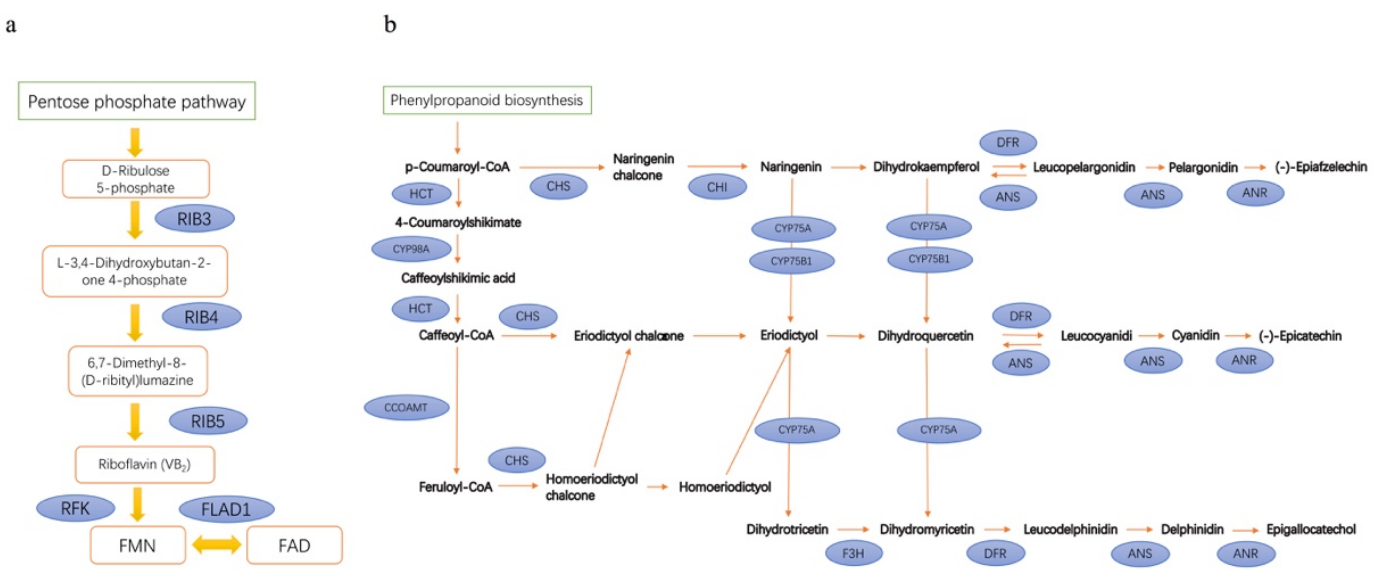
biosynthesis pathways. Genes identified as being involved in two pathways shown in the blue circle.

248 FLAD1: FMN adenylyltransferase; RIB3: 3,4-dihydroxy 2-butanone 4-phosphate synthase; RIB4: 6,7-dimethyl-8-ribityllumazine synthase; RIB5: riboflavin synthase; RFK: riboflavin 5'-phosphotransferase; ANR: anthocyanidin reductase; ANS: leucoanthocyanidin dioxygenase; CCOAMT: caffeoyl-CoA O-methyltransferase; CHI: chalcone isomerase; CHS: chalcone synthase; CYP75A: flavonoid 3',5'hydroxylase; CYP75B1: flavonoid 3'-monooxygenase; CYP98A: coumaroylquinate 3'-monooxygenase; DFR: flavanone 4-reductase; F3H: naringenin 3-dioxygenase; HCT: shikimate O-hydroxycinnamoyltransferase.

\section{Discussion}

This study presents the first draft genome in the Oxalidaceae family. The sequenced species $A$. carambola (star fruit) is a widely cultivated and utilized as an edible fruit and serves as an important source of minerals, vitamins, and phytomedicinal properties. The genome size was assembled to be $470.51 \mathrm{Mb}$ with scaffold N50 of $2.76 \mathrm{Mb}$. Since we cannot compare the genome size with other species in this family, we have found a similar genome size, which is $434.29 \mathrm{Mb}$ of Populus trichocarpa and $350.62 \mathrm{Mb}$ of

264 Ricinus communis in the closest order Malpighiales. However, the genome of the 265 chromosome level will be further required to better understand the diploid character of 266 this species.

267 Totally, 24,726 gene models were identified from $A$. carambola. The gene number 268 is relatively smaller than the earlier reports in A. thaliana, P. trichocarpa, or T. cacao. 269 The length distribution of exons of prediction star fruit proteins was consistent with 
other species, although its intron and CDS length tended to be shorter than other species in the comparison (Supplementary Fig. 3). The proportion of predicted genes that contain InterPro functional domain is $52.3 \%$, and that can be aligned with NCBI nr database $(66.4 \%)$ are the highest among all databases. It is likely that $A$. carambola is so far the only species which has been assembled in the Oxalidaceae family; there might be some evolutionarily unique genes in this family remaining to be annotated.

Later we performed gene family analysis together with the other ten species and identified the significant expansion of 888 gene families contains 2,916 unique genes in A. carambola. These genes have significantly enriched ( $\mathrm{P}$-value $<=0.05$ ) within 28 GO classes, including 18 biological processes, 2 cellular components, and 8 molecular functions (Supplementary Table 6). The DNA binding biological process contained the most genes (60) within the expanded families. Within the significantly enriched biological processes, defense response to fungus might be related to the anti-microbial property of star fruits in the previous studies ${ }^{1}$. On the other hand, we found that oxidoreductase activity is enriched in the molecular function GO class, which could be one of the potential reasons for the antioxidant activity in star fruits.

The genome evolution analysis indicated that star fruit only shared an ancient $\gamma$ event, and no recent WGD was observed. In the genome evolution study of poplar which belongs to Malpighiales, the presence of a hexaploidization event and recent duplication have been reported ${ }^{21}$. This result could partially explain why star fruit has half of the gene sets compared to poplar.

Moreover, within the enriched KEGG pathway, we identified the enzymes involved in nutrition metabolic pathways - vitamin $C$, vitamin $B_{2}$, oxalate, and flavonoid pathways. Although the potential functional enzymes have been identified from the genome, those functional pathways should be verified by the experimental studies in the future.

In the summary, it can be expected that this draft genome will facilitate in understanding the formation of specific and important traits in the star-fruit plant, such as the discovery of biosynthesis pathways of pharmacologically active metabolites, and in the improvement of breeding of strategies of the star-fruit plant.

\section{Materials and methods}

\section{Plant materials and sequencing}

The leaf samples of $A$. carambola were collected from the Ruili Botanical Garden, Yunnan, China. The genomic DNA was extracted by using CTAB (cetyltriethylammonium bromide) method ${ }^{36}$. The $10 \mathrm{X}$ Genome sequencing was performed to obtain high-quality reads. The high-molecular-weight (HMW) DNA was loaded onto a Chromium Controller chip with 10X Chromium reagents and gel beads, and the rest of the procedures were carried out as per the manufacturer's protocol ${ }^{37}$. Then, BGISEQ-500 platform to produce $2 \times 100 \mathrm{bp}$ paired-end sequences, generating a total 
310 of $183 \mathrm{~Gb}$ raw data. The raw reads were filtered using SOAPfilter v2.2 with the 311 following parameters "-q 33 -i 600 -p -1 -f -z -g 1 -M 2 -Q 20”. After filtering low312 quality reads, around $157 \mathrm{~Gb}$ of clean data (high-quality reads $>\mathrm{Q} 35$ ) remained for the 313 next step.

\section{Estimation of $A$. carambola genome size}

316 All the $157 \mathrm{~Gb}$ clean reads obtained from the BGISEQ-500 platform were subjected to 317 the $17 \mathrm{kmer}$ frequency distribution analysis with Jellyfish ${ }^{38}$ using the parameters " $-\mathrm{k}$ 31817 -t 24". The frequency graph was drawn, and the A. carambola genome size was calculated using the formula: genome size $=$ k-mer_Number/Peak_Depth.

\section{De novo genome assembly}

322 The Linked-Read data were assembled using the Supernova v2.1.1 software ${ }^{13}$ using 323 the "--localcores=24 --localmem=350 --max reads 280000000 " parameter. In order to 324 fill the scaffold gaps, GapCloser version $1.12{ }^{39}$ was used with the parameters "-t 12 -1 325 155". Finally, the total assembled length of $A$. carambola genome was $470.51 \mathrm{Mb}$, with a scaffold N50 of $275.76 \mathrm{~Kb}$ and contig N50 of $44.84 \mathrm{~Kb}$, respectively.

\section{Repeat annotation}

329 For the transposable element annotation, RepeatMasker v3.3.0 ${ }^{40}$ and

330 RepeatProteinMasker v3.3.0 ${ }^{40}$ were used against Repbase v16.10 ${ }^{41}$ to identify

331 known repeats in the $A$. carambola genome. Tandem repeats were identified using

332 software Tandem Repeats Finder $v 4.07 \mathrm{~b}^{42}$. De novo repeat identification was

333 conducted using RepeatModeler v1.0.5 ${ }^{43}$ and LTR_FINDER v1.05 ${ }^{44}$ programs,

334 followed by RepeatMasker v3.3.0 ${ }^{40}$ to obtain the final results.

\section{Gene prediction}

337 Prior to the gene prediction analysis, we masked the repetitive regions of the genome. 338 The MAKER-P v2.31 ${ }^{45}$ was utilized to predict the protein-coding genes based on 339 homologous, and de novo prediction evidence. For homologous-based prediction, 340 protein sequences of Theobroma cacao, Prunus persica, Prunus mume, Prunus avium, 341 Populus trichocarpa, Populus euphratica, and Arabidopsis thaliana were aligned 342 against $A$. carambola genome using BLAT ${ }^{46}$. Then, the gene structure was predicted 343 using GeneWise ${ }^{47}$. In order to optimize different ab initio gene predictors, we 344 constructed a series of training set for de novo prediction evidence. Complete gene 345 models by homologous evidence were picked for training in Augustus tool ${ }^{48}$. 346 Genemark-ES v4.21 ${ }^{49}$ was self-trained using the default criteria. The first round of 347 MAKER-P was also run using the default parameters on the basis of above evidences, 348 with the exception for "protein2genome" which was set to " 1 ", yielding only protein349 supported gene models. SNAP ${ }^{50}$ was then trained with these gene models. Default 350 parameters were used to run the second and the final rounds of MAKER-P, generating 351 the final gene models. 


\section{Functional annotation}

354 The predicted gene models were functionally annotated by aligning their protein sequences against the Kyoto Encyclopedia of Genes and Genomes (KEGG) ${ }^{51}$, the Clusters of Orthologous Groups (COG) ${ }^{16}$, SwissProt ${ }^{17}$, TrEMBL, and the National Center for Biotechnology Information (NCBI) non-redundant (NR) protein databases with BLASTP (E-value $<=1 \mathrm{e}-05$ ). Protein motifs and domains were identified by comparing the sequences against various domain databases, including PFAM, PRINTS, PANTHER, ProDom, PROSITE, and SMART using InterProScan v5.21 ${ }^{18}$. For ncRNA annotation, tRNA genes were identified by tRNAscan-SE v1.23 52. For the identification of rRNA genes, we aligned the assembled data against the rRNA sequences of $A$. thaliana using BLASTN (E-value $<=1 \mathrm{e}-05$ ). The miRNAs and snRNAs were predicted by using INFERNAL ${ }^{53}$ software against the Rfam database ${ }^{54}$.

To classify the NBS domain in the star fruit protein sequences, we used hidden Markov models (HMM) to search for the Pfam NBS (NB-ARC) family PF00931 with E-value cutoff of 1.0. To detect TIR domains, the amino acid sequences were also screened by HMM model Pfam TIR PF01582 (E-value $<=1.0$ ). Moreover, to identify LRR motifs, we performed the HMM search for Pfam LRR models (E-value $<=1.0$ ) against star fruit NBS-encoding amino acid sequences.

In order to compare the ortholog genes in vitamin $\mathrm{C}$, vitamin $\mathrm{B}_{2}$, flavonoid and oxalate pathways between other plant species (P. trichocarpa, C. sinensis, A. thaliana, $S$. lycopersicum, C. papaya, and $V$. vinifera), we also annotated the protein sequences by aligning them against the KEGG database ${ }^{51}$ with BLASTP (E-value $<=1 \mathrm{e}-05$ ) and then filtered by Pfam domains which annotated using InterProScan v5.21 ${ }^{18}$.

\section{Genome duplication}

378 The genome sequences for orthologous analysis were downloaded from NCBI 379 (https://www.ncbi.nlm.nih.gov) for A. thaliana (GCA_000001735.2), V. vinifera 380 (GCA_000003745.2), and P. trichocarpa (GCA_000002775.3). Next, we used wgd 381 software 55 to perform the Ks distribution analysis. The paralogous gene families and 382 one-to-one orthologs were performed using "wgd mcl" command. Then, a Ks 383 distribution for a set of paralogous families or one-to-one orthologs was constructed 384 using the "wgd ksd". Next, we performed "wgd kde" for fitting kernel density estimates 385 (KDEs). Finally, the colinear blocks of $A$. carambola paralog were identified by I386 ADHoRe ${ }^{56}$, and colored by their median Ks value.

\section{Gene family construction and phylogenetic analysis}

389 For gene family analysis, the OrthoMCL ${ }^{23}$ software was utilized to construct 390 orthologous gene families on all the protein-coding genes of A. carambola and 12 391 sequenced plant species (B. napus, G. raimondii, H. brasiliensis, J. curcas, $L$. 392 usitatissimum, M. esculenta, P. euphratica, P. trichocarpa, P. mume, R. communis, $T$. 393 cacao, $V$. vinifera). Before OrthoMCL, the BLASTP was used to find similar matches 
394 from different species with an E-value cutoff of 1e-05. The composition of the 395 OrthoMCL clusters was used to calculate the total number of gene families.

396 Orthogroups that were single copy in all species analyzed were selected and aligned 397 using MAFFT v7.310 ${ }^{57}$. Each gene tree was constructed by RAxML v8.2.4 ${ }^{58}$ with 398 GTRGAMMA model. To construct the species phylogenetic tree, a coalescent-based 399 method by ASTRAL v4.10.4 ${ }^{59}$, with 100 replicates of multi-locus bootstrapping ${ }^{60}$ were 400 used.

401 The divergence time between A. carambola and other species were estimated using 402 MCMCTREE ${ }^{58}$ with the default parameter. The expansion and contraction of gene 403 family numbers were predicted using the CAFÉ ${ }^{61}$ by employing the phylogenetic tree 404 and gene family statistics.

405 To further perform the phylogenetic analysis for the key enzyme GalDH in vitamin 406 C pathway, we annotated ortholog genes from 6 other plant species using BLASTP with 407 an E-value cutoff of 1e-05 to align coding sequences against KEGG database. In total, 40855 ortholog genes were used to generate phylogenetic tree by using maximum 409 likelihood (ML) method in RAxML v 8.2.4 ${ }^{58}$ and included 20 runs to find an optimal 410 tree using GTRGAMMA substitution model and 100 nonparametric Bootstrap 411 replicates.

\section{Data availability}

413 The data sets generated and analyzed during the current study are available in the 414 CNGB Nucleotide Sequence Archive (CNSA: https://db.cngb.org/cnsa). The raw 415 sequencing data is under ID CNR0066625, and assembly data is under ID 416 CNA0002506. All other data generated or analyzed during this study are included in 417 this published article and its supplementary information files.

418 Acknowledgments

419 This work was supported by funding from the National Key R\&D Program of China 420 (No. 2019YFC1711000), Shenzhen Municipal Government of China (grants 421 JCYJ20170817145512476 and JCYJ20160510141910129), the Guangdong 422 Provincial Key Laboratory of Genome Read and Write (grant 2017B030301011), and 423 the NMPA Key Laboratory for Rapid Testing Technology of Drugs.

\section{Author information}

425 1. State Key Laboratory of Agricultural Genomics, China National GeneBank, BGI-

426 Shenzhen, Shenzhen 518120, China

427 Yan-nan Fan, Sunil Kumar Sahu, Ting Yang, Wei-xue Mu, Jinpu Wei, Xun Xu, $428 \quad$ Xin Liu \& Huan Liu 
2. BGI-Yunnan, BGI-Shenzhen, Kunming, 650106, China Le Cheng \& Jin-long Yang

3. Forestry Bureau of Ruili, Yunnan Dehong, Ruili 678600, China

4. Department of Biology, University of Copenhagen, Copenhagen, Denmark. Huan Liu

5. Chinese academy of forestry, Beijing, China Yu-xian Zhao

6. Guangdong Provincial Key Laboratory of Genome Read and Write Xun Xu

\section{Contributions}

440 R.-c.M., J.L., J.-m.Z., T.Y., Y.-x.Z. and W.-x.M. collected the samples; W.-x.M and 441 S.K.S. conceived and conducted the experiments; Y.-n.F. and T.Y. analyzed the results; Y.-n.F. and S.K.S wrote the manuscript.

\section{Corresponding authors}

445 Correspondence to Huan Liu liuhuan@genomics.cn

\section{Conflict of interest}

448 The authors declare that they have no conflict of interest.

\section{References}

$4501 \quad$ Muthu, N., Lee, S. Y., Phua, K. K. \& Bhore, S. J. Nutritional, Medicinal and Toxicological 451 Attributes of Star-Fruits (Averrhoa carambola L.): A Review. Bioinformation 12, 420-424, 452 doi:10.6026/97320630012420 (2016).

4532 Khoo, H. et al. A review on underutilized tropical fruits in Malaysia. Guangxi Agricultural $454 \quad$ Sciences 41, 698-702 (2010).

4553 Ray, P. K. Breeding Tropical and Subtropical Fruits. XVI, 338 (Springer-Verlag Berlin $456 \quad$ Heidelberg, 2002).

4574 Wu, S.-C., Wu, S.-H. \& Chau, C.-F. Improvement of the Hypocholesterolemic Activities of 458 Two Common Fruit Fibers by Micronization Processing. Journal of Agricultural and Food Chemistry 57, 5610-5614, doi:10.1021/jf9010388 (2009).

4605 Cabrini, D. A. et al. Analysis of the Potential Topical Anti-Inflammatory Activity of Averrhoa 461 carambola L. in Mice. Evid Based Complement Alternat Med 2011, 908059-908059, 462 doi:10.1093/ecam/neq026 (2011). 
464 chromatography and mass spectrometry. Journal of Chromatography A 1022, 67-75 (2004).

4657 Annegowda, H. V., Bhat, R., Min-Tze, L., Karim, A. A. \& Mansor, S. M. Influence of

466 sonication treatments and extraction solvents on the phenolics and antioxidants in star fruits. $J$ Food Sci Technol 49, 510-514, doi:10.1007/s13197-011-0435-8 (2012).

$468 \quad 8 \quad$ Cazarolli, L. H. et al. Anti-hyperglycemic action of apigenin-6-C- $\beta$-fucopyranoside from $469 \quad$ Averrhoa carambola. Fitoterapia 83, 1176-1183 (2012).

4709 Suluvoy, J. K., Sakthivel, K. M., Guruvayoorappan, C. \& Berlin Grace, V. M. Protective effect of Averrhoa bilimbi L. fruit extract on ulcerative colitis in wistar rats via regulation of inflammatory mediators and cytokines. Biomedicine \& Pharmacotherapy 91, 1113-1121, doi:https://doi.org/10.1016/j.biopha.2017.05.057 (2017). Wei, X. et al. Protective Effects of 2-Dodecyl-6-Methoxycyclohexa-2,5 -Diene-1,4-Dione Isolated from Averrhoa Carambola L. (Oxalidaceae) Roots on Neuron Apoptosis and Memory Deficits in Alzheimer's Disease. Cellular Physiology and Biochemistry 49, 1105-1114, doi:10.1159/000493289 (2018).

Cheng, S. et al. 10KP: A phylodiverse genome sequencing plan. Gigascience 7, giy013 (2018). sequencing of 689 vascular plant species from the Ruili Botanical Garden. GigaScience (2019). Weisenfeld, N. I., Kumar, V., Shah, P., Church, D. M. \& Jaffe, D. B. Direct determination of diploid genome sequences. Genome research 27, 757-767 (2017). phylogenomics. Molecular biology and evolution 35, 543-548 (2017). arXiv preprint arXiv:1303.3997 (2013). genome-scale analysis of protein functions and evolution. Nucleic Acids Res 28, 33-36 (2000). Bairoch, A. \& Apweiler, R. The SWISS-PROT protein sequence database and its supplement TrEMBL in 2000. Nucleic acids research 28, 45-48 (2000). Quevillon, E. et al. InterProScan: protein domains identifier. Nucleic acids research 33, W116-W120 (2005). Argout, X. et al. The genome of Theobroma cacao. Nature genetics 43, 101, doi:10.1038/ng.736 (2011). Swarbreck, D. et al. The Arabidopsis Information Resource (TAIR): gene structure and function annotation. Nucleic Acids Research 36, D1009-D1014, doi:10.1093/nar/gkm965 (2007). Tuskan, G. A. et al. The genome of black cottonwood, Populus trichocarpa (Torr. \& Gray). science 313, 1596-1604, doi:10.1126/science.1128691 (2006). Jaillon, O. et al. The grapevine genome sequence suggests ancestral hexaploidization in major angiosperm phyla. nature 449, 463 (2007). Li, L., Stoeckert, C. J. \& Roos, D. S. OrthoMCL: identification of ortholog groups for 
eukaryotic genomes. Genome research 13, 2178-2189 (2003).

25 Jia, X., Xie, H., Jiang, Y. \& Wei, X. Flavonoids isolated from the fresh sweet fruit of Averrhoa Chase, M. W. et al. An update of the Angiosperm Phylogeny Group classification for the orders and families of flowering plants: APG IV. Botanical Journal of the Linnean Society 181, 1-20 (2016).

26 Moresco, H. H., Queiroz, G. S., Pizzolatti, M. G. \& Brighente, I. M. Chemical constituents carambola, commonly known as star fruit. Phytochemistry 153, 156-162, doi:https://doi.org/10.1016/j.phytochem.2018.06.007 (2018). and evaluation of the toxic and antioxidant activities of Averrhoa carambola leaves. Brazilian Journal of Pharmacognosy 22, 319-324 (2012).

27 Wheeler, G. L., Jones, M. A. \& Smirnoff, N. The biosynthetic pathway of vitamin C in higher plants. Nature 393, 365 (1998)

28 Laing, W. A., Wright, M. A., Cooney, J. \& Bulley, S. M. The missing step of the L-galactose pathway of ascorbate biosynthesis in plants, an L-galactose guanyltransferase, increases leaf ascorbate content. Proceedings of the National Academy of Sciences 104, 9534-9539, doi:10.1073/pnas.0701625104 (2007).

29 Kurup, S. B. \& Mini, S. Averrhoa bilimbi fruits attenuate hyperglycemia-mediated oxidative stress in streptozotocin-induced diabetic rats. journal of food and drug analysis 25, 360-368 (2017).

30 Liu, Y., Zhang, X. \& Tian, X. Extraction and purification of flavonoids in Carambola. Journal of Shenyang Agricultural University 40, 491-493 (2009).

31 Yang, D., Xie, H., Jia, X. \& Wei, X. Flavonoid C-glycosides from star fruit and their antioxidant activity. Journal of Functional Foods 16, 204-210 (2015).

32 Jia, X., Xie, H., Jiang, Y. \& Wei, X. Phytochemistry Flavonoids isolated from the fresh sweet fruit of Averrhoa carambola, commonly known as star fruit. (2018).

33 Moresco, H. H., Queiroz, G. S., Pizzolatti, M. G. \& Brighente, I. Chemical constituents and evaluation of the toxic and antioxidant activities of Averrhoa carambola leaves. Revista Brasileira de Farmacognosia 22, 319-324 (2012).

34 Tiwari, K., Masood, M. \& Minocha, P. Chemical constituents of Gmelina phillipinensis, Adenocalymna nitida, Allamanda cathartica, Averrhoa carambola and Maba buxifolia. Journal of the Indian Chemical Society (1979).

35 Gunasegaran, R. Flavonoids and anthocyanins of three Oxalidaceae. Fitoterapia 63, 89-90 (1992).

Sahu, S. K., Thangaraj, M. \& Kathiresan, K. DNA extraction protocol for plants with high levels of secondary metabolites and polysaccharides without using liquid nitrogen and phenol. ISRN Molecular Biology 2012 (2012). 10X Genomics. https://www.10xgenomics.com., 2017). Marçais, G. \& Kingsford, C. A fast, lock-free approach for efficient parallel counting of occurrences of k-mers. Bioinformatics 27, 764-770 (2011).

39 Luo, R. et al. SOAPdenovo2: an empirically improved memory-efficient short-read de novo assembler. Gigascience 1, 18 (2012).

Tarailo-Graovac, M. \& Chen, N. Using RepeatMasker to identify repetitive elements in 
genomic sequences. Current protocols in bioinformatics 25, 4.10. 11-14.10. 14 (2009). Jurka, J. et al. Repbase Update, a database of eukaryotic repetitive elements. Cytogenetic and genome research 110, $462-467$ (2005). research 27, 573-580 (1999). genomes. Bioinformatics 21, i351-i358 (2005). retrotransposons. Nucleic acids research 35, W265-W268 (2007). MAKER and MAKER-P. Current Protocols in Bioinformatics 48, 4.11. 11-14.11. 39 (2014). Birney, E., Clamp, M. \& Durbin, R. GeneWise and genomewise. Genome research 14, 988995 (2004). a generalized hidden Markov model that uses hints from external sources. $B M C$ bioinformatics 7, 62 (2006). Lomsadze, A., Ter-Hovhannisyan, V., Chernoff, Y. O. \& Borodovsky, M. Gene identification in novel eukaryotic genomes by self-training algorithm. Nucleic acids research 33, 6494-6506 (2005). Kanehisa, M. \& Goto, S. KEGG: kyoto encyclopedia of genes and genomes. Nucleic acids research 28, 27-30 (2000). Lowe, T. M. \& Eddy, S. R. tRNAscan-SE: a program for improved detection of transfer RNA genes in genomic sequence. Nucleic acids research 25, 955-964 (1997). Nawrocki, E. P., Kolbe, D. L. \& Eddy, S. R. Infernal 1.0: inference of RNA alignments. Bioinformatics 25, 1335-1337 (2009). Nawrocki, E. P. et al. Rfam 12.0: updates to the RNA families database. Nucleic acids research 43, D130-D137 (2014). Zwaenepoel, A. \& Van de Peer, Y. wgd—simple command line tools for the analysis of ancient whole-genome duplications. Bioinformatics 35, 2153-2155, doi:10.1093/bioinformatics/bty915 (2018). Proost, S. et al. i-ADHoRe 3.0-fast and sensitive detection of genomic homology in extremely large data sets. Nucleic acids research 40, e11-e11 (2011). improvements in performance and usability. Molecular biology and evolution 30, 772-780 (2013).

$588 \quad 60 \quad$ Seo, T.-K. Calculating bootstrap probabilities of phylogeny using multilocus sequence data. 
bioRxiv preprint doi: https://doi.org/10.1101/851790; this version posted January 28,2020 . The copyright holder for this preprint (which

was not certified by peer review) is the author/funder. All rights reserved. No reuse allowed without permission.

Molecular biology and evolution 25, 960-971 (2008).

59061 De Bie, T., Cristianini, N., Demuth, J. P. \& Hahn, M. W. CAFE: a computational tool for the 591 study of gene family evolution. Bioinformatics 22, 1269-1271 (2006).

592 